\title{
A Complete Parametric Solutions of Eigenstructure Assignment by State-Derivative Feedback for Linear Control Systems
}

\author{
T. H. S. Abdelaziz, M. Valášek
}

In this paper we introduce a complete parametric approach for solving the problem of eigenstructure assignment via state-derivative feedback for linear systems. This problem is always solvable for any controllable systems iff the open-loop system matrix is nonsingular. In this work, two parametric solutions to the feedback gain matrix are introduced that describe the available degrees of freedom offered by the state-derivative feedback in selecting the associated eigenvectors from an admissible class. These freedoms can be utilized to improve robustness of the closed-loop system. Accordingly, the sensitivity of the assigned eigenvalues to perturbations in the system and gain matrix is minimized. Numerical examples are included to show the effectiveness of the proposed approach.

Keywords: eigenstructure assignment, state-derivative feedback, linear control systems, feedback stabilization, parametrization.

\section{Introduction}

Eigenstructure assignment is one of the basic techniques for designing linear control systems. The eigenstructure assignment problem is the problem of assigning both a given self-conjugate set of eigenvalues and the corresponding eigenvectors. Assigning the eigenvalues allows one to alter the stability characteristics of the system, while assigning eigenvectors alters the transient response of the system. Eigenstructure assignment via state feedback has developed the design methods for a wide class of linear systems under full-state feedback with the objective of stabilizing the control systems. The parametric solution of eigenstructure assignment for state feedback has been studied by many researchers [6-10]. Fahmy and Tantawy [7] and Fahmy and O'Reilly [8-9] have developed solutions to the eigenstructure assignment problem. A parametric characterization of the assignable eigenvalues and generalized eigenvectors is presented. Duan [6] presented two complete parametric approaches for eigenstructure assignment in linear systems via state feedback. This methodology is deeply utilized in this research work.

This paper focuses on a special feedback using only state derivatives instead of full-state feedback. Therefore this feedback is called state-derivative feedback. The problem of arbitrary eigenstructure assignment using full-state derivative feedback naturally arises. The motivation for state derivative feedback comes from controlled vibration suppression of mechanical systems. The main sensors of vibration are accelerometers. From accelerations we can reconstruct velocities with reasonable accuracy, but not the displacements. Therefore the available signals for feedback are accelerations and velocities only, and these are exactly the derivatives of states of the mechanical systems, which are the velocities and displacements. Direct measurement of the state is difficult to achieve. One necessary condition for a control strategy to be implementable is that it must use the available measured responses to determine the control action. All of the previous research in control has assumed that all of the states can be directly measured (i.e., that there is full-state feedback).
Many papers have been published on controlling this class of systems, (e.g. [12-17]) describing the acceleration feedback for controlled vibration suppression. However, the eigenstructure assignment approach for feedback gain determination has not been used at all or has not been solved generally. Other papers dealing with acceleration feedback for mechanical systems are [18-19], but here the feedback uses all states (positions, velocities) and accelerations additionally.

Abdelaziz and Valasek [1-3] have recently presented an eigenvalue assignment technique via state-derivative feedback for single-input and multi-input time-invariant linear systems. Eigenstructure assignment via state-derivative feedback is introduced in [4-5]. In this paper, two complete parametric approaches for eigenstructure assignment in linear systems via state-derivative feedback are proposed. Two complete parametric expressions for the closed-loop eigenvector matrices and the feedback gains are established in terms of closed-loop eigenvalues and a group of parameter vectors. Both the closed-loop eigenvalues and this group of parameters can be properly chosen to produce a closed-loop system with some additional desired system specifications. The necessary and sufficient conditions for the existence of the eigenstructure assignment problem are described. The proposed controller is based on the measurement and feedback of the state derivatives of the system. This work has successfully extended previous techniques by state feedback and has modified them to state-derivative feedback. Finally, numerical examples are included to demonstrate the effectiveness of this approach. The main contribution of this work is an efficient technique that solves the eigenstructure assignment problem via state-derivative feedback systems. The procedure defined here represents a unique treatment for extending the eigenstructure assignment technique using the state-derivative feedback in the literature.

This paper is organized as follows. In the next section, the problem formulation and the necessary and sufficient conditions for the existence of the eigenstructure assignment problem are described. Additionally, two complete paramet- 
ric solutions to the eigenstructure assignment problem via state-derivative feedback are presented. In section 3, illustrative examples are presented. Finally, conclusions are discussed in section 4 .

\section{Eigenstructure assignment by state-derivative feedback for time-invariant systems}

In this section, we present two complete parametric approaches for solving the eigenstructure assignment problem via state-derivative feedback for linear time-invariant systems.

\subsection{Eigenstructure assignment problem formulation}

Consider a linear, time-invariant, completely controllable system

$$
\dot{\boldsymbol{x}}(t)=\boldsymbol{A x}(t)+\boldsymbol{B} \boldsymbol{u}(t), \boldsymbol{x}\left(t_{0}\right)=\boldsymbol{x}_{0},
$$

where $\dot{\boldsymbol{x}}(t) \in \mathrm{R}^{n}, \boldsymbol{x}(t) \in \mathrm{R}^{n}$ and $\boldsymbol{u}(t) \in \mathrm{R}^{m}$ are the state-derivative, the state and the control input vectors, respectively, $(m \leq n)$, while $\boldsymbol{A} \in \mathrm{R}^{n \times n}$ and $\boldsymbol{B} \in \mathrm{R}^{n \times m}$ are the system and control gain matrices, respectively. The fundamental assumption imposed on the system is that the system is completely controllable and matrix $\boldsymbol{B}$ has a full column rank $m$.

The objective is to stabilize the system by means of a linear feedback that enforces the desired characteristic behavior for the states. The problem is to find the state-derivative feedback control law

$$
\boldsymbol{u}(t)=-\boldsymbol{K} \dot{\boldsymbol{x}}(t),
$$

which assigns prescribed closed-loop eigenvalues and corresponding eigenvectors that stabilize the system and achieve the desired performance. Here, the first derivative vector of state-space $\dot{x}(t)$ is utilized instead of the vector of state-space $x(t)$.

Then, the closed-loop system dynamics becomes

$$
\dot{\boldsymbol{x}}(t)=\left(\boldsymbol{I}_{n}+\boldsymbol{B} \boldsymbol{K}\right)^{-1} \boldsymbol{A} \boldsymbol{x}(t),
$$

where $\boldsymbol{I}_{n}$ is the $n \times n$ identity matrix. In what follows, we assume that $\left(\boldsymbol{I}_{n}+\boldsymbol{B K}\right)$ has a full rank in order that the closed-loop system is well defined.

The closed-loop characteristic polynomial is given by

$$
\operatorname{det}=\left[\lambda \boldsymbol{I}_{n}-\left(\boldsymbol{I}_{n}+\boldsymbol{B} \boldsymbol{K}\right)^{-1} \boldsymbol{A}\right]=0 \text {. }
$$

Let $\Gamma=\left\{\lambda_{i}, \lambda_{i} \in \mathrm{C}, i=1, \ldots, s, 1 \leq s<n\right\}$ be a set of desired self-conjugate eigenvalues, where $s$ is the number of distinct eigenvalues, and denote the algebraic and geometric multiplicity of the $i$ th eigenvalue $\lambda_{i}$ by $m_{i}$ and $q_{i}$, respectively, $\left(1 \leq q_{i} \leq m_{i}\right)$. The length of $q_{i}$ chains of generalized eigenvectors with $\lambda_{i}$ are denoted by $p_{i j},\left(j=1, \ldots, q_{i}\right)$. Then in the Jordan canonical form of the closed-loop matrix, there are $q_{i}$ blocks associated with the $i$ th eigenvalue $\lambda_{i}$ of orders $p_{i j}$. It is satisfying that

$$
\sum_{i=1}^{s} \sum_{j=1}^{q_{i}} p_{i j}=n
$$

In this work, we restrict ourselves by $m_{i}=q_{i}$. This means that the multiple eigenvalues are not split; they are placed in one Jordan block. The partial multiplicities are not placed. Therefore, the Rosenbrock's inequalities are fulfilled.
The right eigenvector and generalized eigenvectors of the closed-loop matrix with $\lambda_{i}$ are denoted by $\mathbf{v}_{i j}^{k} \in \mathrm{C}^{n}, i=1, \ldots, s$, $j=1, \ldots, q_{i}, k=1, \ldots, p_{i j}$. According to the definition of the right eigenvector and generalized eigenvectors for multiple eigenvalues, then

$\left(\left(\boldsymbol{I}_{n}+\boldsymbol{B} \boldsymbol{K}\right)^{-1} \boldsymbol{A}-\lambda_{i} \boldsymbol{I}_{n}\right) \mathbf{v}_{i j}^{k}=\mathbf{v}_{i j}^{k-1}, \mathbf{v}_{i j}^{0}=\mathbf{0}, \forall i, j, k$.

This equation demonstrates the relation of assignable right generalized eigenvectors with the associated eigenvalue. The notations are defined as

$$
\begin{aligned}
& \boldsymbol{V} \equiv\left[\boldsymbol{V}_{1}, \ldots, \boldsymbol{V}_{s}\right] \in \mathrm{C}^{n \times n}, \\
& \boldsymbol{V}_{i} \equiv\left[\boldsymbol{V}_{i 1}, \ldots, \boldsymbol{V}_{i q_{i}}\right] \in \mathrm{C}^{n \times m_{i}}, \\
& \boldsymbol{V}_{i j} \equiv\left[\mathbf{v}_{i j}^{1}, \ldots, \mathbf{v}_{i j}^{p_{i j}}\right] \in \mathrm{C}^{n \times p_{i j}},
\end{aligned}
$$

where $V_{i}$ contains all right eigenvectors and generalized eigenvectors associated with the eigenvalue $\lambda_{i}$, and $\operatorname{det}(\boldsymbol{V}) \neq 0$.

Then, the eigenstructure assignment problem for system (1) via state-derivative feedback can be stated as follows:

\section{Eigenstructure assignment problem:}

Given the real pair $(\boldsymbol{A}, \boldsymbol{B})$ and the desired self-conjugate set $\left\{\lambda_{1}, \ldots, \lambda_{n}\right\} \in \mathrm{C}$, find the real state-derivative feedback gain matrix $\boldsymbol{K} \in \mathrm{R}^{m \times n}$ that will make the closed-loop matrix $\left(\boldsymbol{I}_{n}+\boldsymbol{B} \boldsymbol{K}\right)^{-1} \boldsymbol{A}$ have admissable eigenvalues and the associated set of right eigenvector and generalized eigenvector matrix $\boldsymbol{V}$.

The necessary and sufficient conditions that ensure solvability of the eigenstructure assignment problem via state-derivative feedback are presented in the following lemma.

\section{Lemma 1:}

The eigenstructure assignment problem for the real pair $(\boldsymbol{A}, \boldsymbol{B})$ is solvable for any arbitrary nonzero, self-conjugate, closed-loop poles, if $(\boldsymbol{A}, \boldsymbol{B})$ is completely controllable, that is

$$
\begin{aligned}
& \operatorname{rank}\left[\boldsymbol{B}, \boldsymbol{A B}, \ldots, \boldsymbol{A}^{n-1} \boldsymbol{B}\right]=n, \\
& \text { or } \operatorname{rank}\left[\lambda \boldsymbol{I}_{n}-\boldsymbol{A}, \boldsymbol{B}\right]=n, \\
& \forall \lambda \in \mathrm{C},
\end{aligned}
$$

and $\boldsymbol{A}$ is nonsingular.

Proof: From this condition, the closed-loop matrix must be defined. This means the matrix $\left(\boldsymbol{I}_{n}+\boldsymbol{B} \boldsymbol{K}\right)$ is of full rank and $\operatorname{det}\left(\boldsymbol{I}_{n}+\boldsymbol{B} \boldsymbol{K}\right) \neq 0$. Then, from (5) it can easily be rewritten as

$$
\left(\boldsymbol{I}_{n}+\boldsymbol{B} \boldsymbol{K}\right)^{-1} \boldsymbol{A} \boldsymbol{V}=\boldsymbol{V} \boldsymbol{\Lambda}
$$

where $\boldsymbol{\Lambda} \in \mathrm{C}^{n \times n}$ is in Jordan canonical form with the desired eigenvalues on the diagonal. Then

$$
A V-V \Lambda=B K V \Lambda
$$

which can be written as

$$
\boldsymbol{I}_{n}+\boldsymbol{B} \boldsymbol{K}=\boldsymbol{A} \boldsymbol{V} \boldsymbol{\Lambda}^{-1} \boldsymbol{V}^{-1} \text {. }
$$

Then,

$$
\operatorname{det}\left(\boldsymbol{I}_{n}+\boldsymbol{B} \boldsymbol{K}\right)=\operatorname{det}\left(\boldsymbol{A} \boldsymbol{V} \boldsymbol{\Lambda}^{-1} \boldsymbol{V}^{-1}\right)=\operatorname{det}(\boldsymbol{A}) \operatorname{det}\left(\boldsymbol{\Lambda}^{-1}\right) \neq 0 .
$$

Since $V$ must be nonsingular, then $\operatorname{det}(\boldsymbol{A}) \neq 0$ and $\operatorname{det}(\boldsymbol{\Lambda}) \neq 0$. Therefore, matrices $\boldsymbol{A}$ and $\boldsymbol{\Lambda}$ should be of full rank in order for the closed-loop matrix to be defined.

Thus, the necessary and sufficient conditions for existence of the solution to the eigenstructure assignment problem via state-derivative feedback is that the system is completely con- 
trollable and all eigenvalues of the original system are nonzero ( $\boldsymbol{A}$ has full rank). We remark that the requirement that matrix $\boldsymbol{\Lambda}$ is diagonal, together with the invertibility of $\boldsymbol{V}$, ensures that the closed-loop system is non-defective. Non-defective systems are desirable because the poles of such systems are less sensitive to system parameter perturbation [10].

Based on the above necessary and sufficient conditions, two parametric forms are derived for the state-derivative feedback gain matrix $\boldsymbol{K}$ that assigns the desired closed-loop poles.

\subsection{Eigenstructure assignment}

The main work now is to find a parametric solution to the state-derivative feedback gain matrix $\boldsymbol{K}$ that assigns the desired closed-loop eigenvalues and associated eigenvectors.

Equation (5) can be rewritten as

$A \mathbf{v}_{i j}^{k}=\lambda_{i}\left(\boldsymbol{I}_{n}+\boldsymbol{B} \boldsymbol{K}\right) \mathbf{v}_{i j}^{k}+\left(\boldsymbol{I}_{n}+\boldsymbol{B} \boldsymbol{K}\right) \mathbf{v}_{i j}^{k-1}, \mathbf{v}_{i j}^{0}=\boldsymbol{0}, \forall i, j, k$.

Let the auxiliary vectors

$\mathbf{w}_{i j}^{k}=K_{\mathbf{v}}^{k} \in \mathrm{C}^{m}, i=1, \ldots, s, j=1, \ldots, q_{i}, k=1, \ldots, p_{i j}$,

be introduced. The set of $\mathbf{w}_{i j}^{k}$ is defined in a similar manner to the set of $\mathbf{v}_{i j}^{k}$ as follows,

$$
\begin{aligned}
& \boldsymbol{W} \equiv\left[\boldsymbol{W}_{1}, \ldots, \boldsymbol{W}_{s}\right] \in \mathrm{C}^{m \times n}, \\
& \boldsymbol{W}_{i} \equiv\left[\boldsymbol{W}_{i 1}, \ldots, \boldsymbol{W}_{i q_{i}}\right] \in \mathrm{C}^{m \times m_{i}}, \\
& \boldsymbol{W}_{i j} \equiv\left[\mathbf{w}_{i j}^{1}, \ldots, \mathbf{w}_{i j}^{p_{i j}}\right] \in \mathrm{C}^{m \times p_{i j}} .
\end{aligned}
$$

This leads to

$\left(\lambda_{i} \boldsymbol{I}_{n}-\boldsymbol{A}\right) \mathbf{v}_{i j}^{k}+\lambda_{i} \boldsymbol{B} \mathbf{w}_{i j}^{k}=-\mathbf{v}_{i j}^{k-1}-\boldsymbol{B} \mathbf{w}_{i j}^{k-1}$,

$\mathbf{v}_{i j}^{0}=\boldsymbol{0}, \mathbf{w}_{i j}^{0}=\boldsymbol{0}, \forall i, j, k$.

The above equation can be equivalently written in the following compact matrix form,

$\left[\lambda_{i} \boldsymbol{I}_{n}-\boldsymbol{A}, \lambda_{i} \boldsymbol{B}\right]\left(\begin{array}{c}\mathbf{v}_{i j}^{k} \\ \mathbf{w}_{i j}^{k}\end{array}\right)=-\left[\boldsymbol{I}_{n}, \boldsymbol{B}\right]\left(\begin{array}{c}\mathbf{v}_{i j}^{k-1} \\ \mathbf{w}_{i j}^{k-1}\end{array}\right)$,

$\mathbf{v}_{i j}^{0}=\boldsymbol{0}, \mathbf{w}_{i j}^{0}=\boldsymbol{0}, \forall i, j, k$.

Finally, the parametric equation to the right eigenvector and generalized eigenvectors can be expressed as

$\left[\lambda_{i} \boldsymbol{I}_{n}-\boldsymbol{A}, \lambda_{i} \boldsymbol{B}\right] \xi_{i j}^{k}=-\left[\boldsymbol{I}_{n}, \boldsymbol{B}\right] \xi_{i j}^{k-1}$,

$\xi_{i j}^{k}=\left(\begin{array}{c}\mathbf{v}_{i j}^{k} \\ \mathbf{w}_{i j}^{k}\end{array}\right), \xi_{i j}^{0}=\boldsymbol{0}, \forall i, j, k$.

Then, parameter vectors $\xi_{i j}^{k} \in \mathrm{C}^{n+m}$ are chosen arbitrary under the condition that the columns of matrix $\boldsymbol{V}$ are linearly independent.

A parametric solution to the eigenstructure assignment problem via state-derivative feedback is derived from (11) as

$$
\boldsymbol{K}=\boldsymbol{W} \boldsymbol{V}^{-1}
$$

where

$\boldsymbol{V}=\left[V_{1}\left(\lambda_{1}\right), \ldots, V_{s}\left(\lambda_{s}\right)\right], \boldsymbol{W}=\left[W_{1}\left(\lambda_{1}\right), \ldots, W_{s}\left(\lambda_{s}\right)\right]$.

Then the feedback gain matrix is parametrized directly in terms of the eigenstructure of the closed-loop system, which can be selected to ensure robustness by exploiting freedom of these parameters.

There exists a real feedback gain matrix $\boldsymbol{K}$ if and only if the following three conditions are satisfied:

1. The assigned eigenvalues are symmetric with respect to the real axis.

2. The right generalized eigenvectors $\left\{\mathbf{v}_{i j}^{k} \in \mathrm{C}^{n}, i=1, \ldots, s, j=1, \ldots, q_{i}, k=1, \ldots, p_{i j}\right\}$

are linearly independent and for complex-conjugate poles, $\bar{\lambda}_{i_{2}}=\lambda_{i_{1}}$ then $\overline{\mathbf{v}}_{i_{2} j}^{k}=\mathbf{v}_{i_{1} j}^{k}$.

3. There exists a set of vectors

$\left\{\mathbf{w}_{i j}^{k} \in \mathrm{C}^{m}, i=1, \ldots, s, j=1, \ldots, q_{i}, k=1, \ldots, p_{i j}\right\}$,

satisfying (13) and $\overline{\mathbf{w}}_{i_{2} j}^{k}=\mathbf{w}_{i_{1} j}^{k}$ for $\bar{\lambda}_{i_{2}}=\lambda_{i_{1}}$.

The parametric formula for the state-derivative feedback gain matrix $\boldsymbol{K}$ that assigns the desired closed-loop poles system is now derived. In the following, we obtain the more general parametric solutions of $\mathbf{v}_{i j}^{k}$ and $\mathbf{w}_{i j}^{k}$ in (13). Two complete parametric forms are introduced and a new procedure is derived, which yields a parametric expression for $\boldsymbol{K}$ involving free parameter vectors.

\subsection{A parametrization approach for eigenstructure assignment}

The aim now is to find a parametric solution to the eigenstructure assignment problem via state-derivative feedback. We remark that the development of parametric solutions to this problem is useful in that one can then think of solving other important variations of the problem, such as the robust eigenstructure assignment problem, by exploiting freedom of these parameters. The relation demonstrating the assignable right generalized eigenvectors with the eigenvalues is (13).

Definition 1: A square polynomial matrix $\boldsymbol{P}(\lambda)$ is called a unimodular matrix if its determinant is a nonzero constant.

Definition 2: polynomial matrix $\boldsymbol{P}(\lambda)$ is a unimodular matrix if and only if $\boldsymbol{P}(\lambda)$ equals the product of some finite number of elementary row (or column) transformation matrices.

It is well known that the matrix pair $(\boldsymbol{A}, \boldsymbol{B})$ is controllable if and only if

$$
\operatorname{rank}\left[\lambda \boldsymbol{I}_{n}-\boldsymbol{A}, \boldsymbol{B}\right]=n, \forall \lambda \in \mathrm{C} .
$$

Due to the controllability of $(\boldsymbol{A}, \boldsymbol{B})$, there exist unimodular matrices $\boldsymbol{P}(\lambda) \in \mathrm{C}^{n \times n}$ and $\boldsymbol{Q}(\lambda) \in \mathrm{C}^{(n+m) \times(n+m)}$ satisfying the following equation:

$$
\boldsymbol{P}(\lambda)\left[\lambda \boldsymbol{I}_{n}-\boldsymbol{A}, \boldsymbol{B}\right] \boldsymbol{Q}(\lambda)=\left[\boldsymbol{0}, \boldsymbol{I}_{n}\right] .
$$

Partition the polynomial matrix $\boldsymbol{Q}(\lambda)$ into the following form

$$
\boldsymbol{Q}(\lambda)=\left(\begin{array}{l}
\boldsymbol{Q}_{1}(\lambda) \\
\boldsymbol{Q}_{2}(\lambda)
\end{array}\right)=\left(\begin{array}{ll}
\boldsymbol{Q}_{11}(\lambda) & \boldsymbol{Q}_{12}(\lambda) \\
\boldsymbol{Q}_{21}(\lambda) & \boldsymbol{Q}_{22}(\lambda)
\end{array}\right)
$$

with $\boldsymbol{Q}_{1}(\lambda) \in \mathrm{C}^{n \times(n+m)}, \quad \boldsymbol{Q}_{2}(\lambda) \in \mathrm{C}^{m \times(n+m)}, \quad \boldsymbol{Q}_{11}(\lambda) \in C^{n \times m}$, $\boldsymbol{Q}_{12}(\lambda) \in \mathrm{C}^{n \times n}, \boldsymbol{Q}_{21}(\lambda) \in \mathrm{C}^{m \times m}$ and $\boldsymbol{Q}_{22}(\lambda) \in \mathrm{C}^{m \times n}$. 
Then, converting (16) to the following form,

$\boldsymbol{P}(\lambda)\left[\lambda \boldsymbol{I}_{n}-\boldsymbol{A}, \lambda \boldsymbol{B}\right]\left(\begin{array}{l}\boldsymbol{Q}_{1}(\lambda) \\ \widetilde{\boldsymbol{Q}}_{2}(\lambda)\end{array}\right)=\left[\boldsymbol{0}, \boldsymbol{I}_{n}\right]$,

with $\widetilde{\boldsymbol{Q}}_{2}(\lambda)=\left(\frac{\boldsymbol{Q}_{2}(\lambda)}{\lambda}\right)$.

Now, the following theorem gives a parametric solution to the eigenstructure assignment problem via state-derivative feedback. The parametric solutions of $\mathbf{v}_{i j}^{k}$ and $\mathbf{w}_{i j}^{k}$ in (13) can now be given.

\section{Theorem 1:}

Let the matrix pair $(\boldsymbol{A}, \boldsymbol{B})$ be controllable, where matrix $\boldsymbol{A} \in \mathrm{R}^{n \times n}$ is nonsingular and matrix $\boldsymbol{B} \in \mathrm{R}^{n \times m}$ has a full column rank $m$. Then all solutions of (13), $\mathbf{v}_{i j}^{k}$ and $\mathbf{w}_{i j}^{k}$, are given by:

$\left(\begin{array}{c}\mathbf{v}_{i j}^{k} \\ \mathbf{w}_{i j}^{k}\end{array}\right)=\left(\begin{array}{c}\boldsymbol{Q}_{1}(\lambda) \\ \widetilde{\boldsymbol{Q}}_{2}(\lambda)\end{array}\right)\left(\begin{array}{c}f_{i j}^{k} \\ \boldsymbol{P}\left(\lambda_{i}\right)\left(-\mathbf{v}_{i j}^{k-1}-\boldsymbol{B} \mathbf{w}_{i j}^{k-1}\right)\end{array}\right)$,

$\mathbf{v}_{i j}^{0}=\boldsymbol{0}, \mathbf{w}_{i j}^{0}=\mathbf{0}$.

Or, equivalently, as

$\mathbf{v}_{i j}^{k}=\boldsymbol{Q}_{11}\left(\lambda_{i}\right) f_{i j}^{k}-\boldsymbol{Q}_{12}\left(\lambda_{i}\right) \boldsymbol{P}\left(\lambda_{i}\right)\left(\mathbf{v}_{i j}^{k-1}+\boldsymbol{B} \mathbf{w}_{i j}^{k-1}\right), \mathbf{v}_{i j}^{0}=\mathbf{0}$

and

$\mathbf{w}_{i j}^{k}=\frac{1}{\lambda_{i}}\left(\boldsymbol{Q}_{21}\left(\lambda_{i}\right) f_{i j}^{k}-\boldsymbol{Q}_{22}\left(\lambda_{i}\right) \boldsymbol{P}\left(\lambda_{i}\right)\left(\mathbf{v}_{i j}^{k-1}+\boldsymbol{B} \mathbf{w}_{i j}^{k-1}\right)\right), \mathbf{w}_{i j}^{0}=\mathbf{0}$ $i=1, \ldots, s, j=1, \ldots, q_{i}, k=1, \ldots, p_{i j}$,

where $\boldsymbol{P}(\lambda)$ and $\boldsymbol{Q}(\lambda)$ are unimodular matrices satisfying (16), and $f_{i j}^{k} \in \mathrm{C}^{m}$ are arbitrarily free parameter vectors satisfying the following two contraints:

$$
\operatorname{det}(\boldsymbol{V}) \neq \boldsymbol{O} \text { and } \bar{f}_{i_{2} j}^{k}=f_{i_{1} j}^{k} \text { if } \overline{\boldsymbol{\lambda}}_{i_{2}}=\lambda_{i_{1}} .
$$

Proof: First, we need to show that the set of vectors satisfying (13) and the set of vectors given by (18) are equal. Then, using (13) and (18), we have

$\left[\lambda_{i} \boldsymbol{I}_{n}-\boldsymbol{A}, \lambda_{i} \boldsymbol{B}\right]\left(\begin{array}{c}\mathbf{v}_{i j}^{k} \\ \mathbf{w}_{i j}^{k}\end{array}\right)=\left[\lambda_{i} \boldsymbol{I}_{n}-\boldsymbol{A}, \lambda_{i} \boldsymbol{B}\right]\left(\begin{array}{c}\boldsymbol{Q}_{1}\left(\lambda_{i}\right) \\ \widetilde{\boldsymbol{Q}}_{2}\left(\lambda_{i}\right)\end{array}\right)$

$\left(\begin{array}{c}f_{i j}^{k} \\ \boldsymbol{P}\left(\lambda_{i}\right)\left(-\mathbf{v}_{i j}^{k-1}-\boldsymbol{B} \mathbf{w}_{i j}^{k-1}\right)\end{array}\right)$

$=\boldsymbol{P}^{-1}\left(\lambda_{i}\right)\left[\boldsymbol{0}, \boldsymbol{I}_{n}\right]\left(\begin{array}{c}f_{i j}^{k} \\ \boldsymbol{P}\left(\lambda_{i}\right)\left(-\mathbf{v}_{i j}^{k-1}-\boldsymbol{B} \mathbf{w}_{i j}^{k-1}\right)\end{array}\right)$

$=-\mathbf{v}_{i j}^{k-1}-\boldsymbol{B} \mathbf{w}_{i j}^{k-1}=-\left[\boldsymbol{I}_{n}, \boldsymbol{B}\right]\left(\begin{array}{c}\mathbf{v}_{i j}^{k-1} \\ \mathbf{w}_{i j}^{k-1}\end{array}\right)$,

$\mathbf{v}_{i j}^{0}=\boldsymbol{0}, \mathbf{w}_{i j}^{0}=\boldsymbol{0}, \forall i, j, k$.

Therefore, the vectors given by (18) satisfy (13). Now, we show that vectors $\mathbf{v}_{i j}^{k}$ and $\mathbf{w}_{i j}^{k}\left(i=1, \ldots, s, j=1, \ldots, q_{i}, k=1\right.$, $\ldots, p_{i j}$ ) satisfying (13) can be expressed in the form of (18). From (17) we can obtain

$\boldsymbol{P}\left(\lambda_{i}\right)\left[\lambda_{i} \boldsymbol{I}_{n}-\boldsymbol{A}, \lambda_{i} \boldsymbol{B}\right]=\left[\boldsymbol{0}, \boldsymbol{I}_{n}\right]\left(\begin{array}{c}\boldsymbol{Q}_{1}\left(\lambda_{i}\right) \\ \widetilde{\boldsymbol{Q}}_{2}\left(\lambda_{i}\right)\end{array}\right)^{-1}, i=1, \ldots, s$.
Then

$\boldsymbol{P}\left(\lambda_{i}\right)\left[\lambda_{i} \boldsymbol{I}_{n}-\boldsymbol{A}, \lambda_{i} \boldsymbol{B}\right]\left(\begin{array}{c}\mathbf{v}_{i j}^{k} \\ \mathbf{w}_{i j}^{k}\end{array}\right)=\left[\boldsymbol{0}, \boldsymbol{I}_{n}\right]\left(\begin{array}{c}\boldsymbol{Q}_{1}\left(\lambda_{i}\right) \\ \widetilde{\boldsymbol{Q}}_{2}\left(\lambda_{i}\right)\end{array}\right)^{-1}\left(\begin{array}{c}\mathbf{v}_{i j}^{k} \\ \mathbf{w}_{i j}^{k}\end{array}\right)$

and

$\boldsymbol{P}\left(\lambda_{i}\right)\left(-\mathbf{v}_{i j}^{k-1}-\boldsymbol{B} \mathbf{w}_{i j}^{k-1}\right)=\left[\boldsymbol{0}, \boldsymbol{I}_{n}\right]\left(\begin{array}{c}f_{i j}^{k} \\ \mathbf{e}_{i j}^{k}\end{array}\right)$,

$\mathbf{v}_{i j}^{0}=\boldsymbol{0}, \mathbf{w}_{i j}^{0}=\mathbf{0}, \forall i, j, k$,

where

$$
\left(\begin{array}{c}
f_{i j}^{k} \\
\mathbf{e}_{i j}^{k}
\end{array}\right)=\left(\begin{array}{c}
\boldsymbol{Q}_{1}\left(\lambda_{i}\right) \\
\widetilde{\boldsymbol{Q}}_{2}\left(\lambda_{i}\right)
\end{array}\right)^{-1}\left(\begin{array}{c}
\mathbf{v}_{i j}^{k} \\
\mathbf{w}_{i j}^{k}
\end{array}\right), \forall i, j, k .
$$

Then from (22) we obtain

$\mathbf{e}_{i j}^{k}=\boldsymbol{P}\left(\lambda_{i}\right)\left(-\mathbf{v}_{i j}^{k-1}-\boldsymbol{B} \mathbf{w}_{i j}^{k-1}\right), \mathbf{v}_{i j}^{0}=\mathbf{0}, \mathbf{w}_{i j}^{0}=\boldsymbol{0}, \forall i, j, k$.

Substituting (24) into (23) we obtain (18).

Assuming zero initial conditions and applying the Laplace transformation to (1), we obtain

$\boldsymbol{X}(\lambda)=\left(\lambda \boldsymbol{I}_{n}-\boldsymbol{A}\right)^{-1} \boldsymbol{B} \boldsymbol{U}(\lambda)=\boldsymbol{G}(\lambda) \boldsymbol{U}(\lambda)$.

Then the behaviour of our linear system is described by a rational matrix function $\left(\lambda \boldsymbol{I}_{n}-\boldsymbol{A}\right)^{-1} \boldsymbol{B}$ of size $n \times m$ of a complex variable $\lambda$. The input-state transfer function of the system can be factorized as

$$
\boldsymbol{G}(\lambda)=\left(\lambda \boldsymbol{I}_{n}-\boldsymbol{A}\right)^{-1} \boldsymbol{B}=\boldsymbol{N}(\lambda) \boldsymbol{D}^{-1}(\lambda),
$$

where $\boldsymbol{N}(\lambda) \in \mathrm{C}^{n \times m}$ and $\boldsymbol{D}(\lambda) \in \mathrm{C}^{m \times m}$ are right coprime polynomial matrices in $\lambda$.

The above equation can be written as

$\left(\lambda \boldsymbol{I}_{n}-\boldsymbol{A}\right) \boldsymbol{N}(\lambda)+\lambda \boldsymbol{B} \widetilde{\boldsymbol{D}}(\lambda)=0$, with $\widetilde{\boldsymbol{D}}(\lambda)=\frac{-\boldsymbol{D}(\lambda)}{\lambda}$.

Now, the following theorem gives a parametric solution to the eigenstructure assignment problem via state-derivative feedback.

\section{Theorem 2:}

Let the matrix pair $(\boldsymbol{A}, \boldsymbol{B})$ be controllable, where matrix $\boldsymbol{A} \in \mathrm{R}^{n \times n}$ is nonsingular and matrix $\boldsymbol{B} \in \mathrm{R}^{n \times m}$ has a full column rank $m$. Then all solutions of (13), $\mathbf{v}_{i j}^{k}$ and $\mathbf{w}_{i j}^{k}$, are given by:

$$
\begin{aligned}
\left(\begin{array}{c}
\mathbf{v}_{i j}^{k} \\
\mathbf{w}_{i j}^{k}
\end{array}\right)= & \left(\begin{array}{l}
\boldsymbol{N}\left(\lambda_{i}\right) \\
\widetilde{\boldsymbol{D}}\left(\lambda_{i}\right)
\end{array}\right) f_{i j}^{k}+\frac{\mathrm{d}}{\mathrm{d} \lambda}\left(\begin{array}{c}
\boldsymbol{N}\left(\lambda_{i}\right) \\
\widetilde{\boldsymbol{D}}\left(\lambda_{i}\right)
\end{array}\right) f_{i j}^{k-1}+\ldots \\
& +\frac{1}{(k-1) !} \frac{\mathrm{d}^{k-1}}{\mathrm{~d} \lambda^{k-1}}\left(\begin{array}{c}
\boldsymbol{N}\left(\lambda_{i}\right) \\
\widetilde{\boldsymbol{D}}\left(\lambda_{i}\right)
\end{array}\right) f_{i j}^{1} .
\end{aligned}
$$

Or, equivalently, as

$$
\begin{aligned}
\mathbf{v}_{i j}^{k} & =\boldsymbol{N}\left(\lambda_{i}\right) f_{i j}^{k}+\sum_{l=1}^{k-l} \frac{1}{l !} \frac{\mathrm{d}^{l}}{\mathrm{~d} \lambda^{l}}\left(\boldsymbol{N}\left(\lambda_{i}\right)\right) f_{i j}^{k-l}, \mathbf{v}_{i j}^{0}=\mathbf{0}, \\
& \text { and } \\
\mathbf{w}_{i j}^{k} & =\widetilde{\boldsymbol{D}}\left(\lambda_{i}\right) f_{i j}^{k}+\sum_{l=1}^{k-l} \frac{1}{l !} \frac{\mathrm{d}^{l}}{\mathrm{~d} \lambda^{l}}\left(\widetilde{\boldsymbol{D}}\left(\lambda_{i}\right)\right) f_{i j}^{k-l}, \mathbf{w}_{i j}^{0}=\mathbf{0}, \\
i & =1, \ldots, s, j=1, \ldots, q_{i}, k=1, \ldots, p_{i j},
\end{aligned}
$$


where $\boldsymbol{N}(\lambda)$ and $\widetilde{\boldsymbol{D}}(\lambda)$ are polynomial matrices satisfying (27) and $f_{i j}^{k} \in \mathrm{C}^{m}$ are arbitrarily free parameter vectors satisfying the following two contraints:

$$
\operatorname{det}(\boldsymbol{V}) \neq \boldsymbol{O} \text { and } \bar{f}_{i_{2} j}^{k}=f_{i_{1} j}^{k} \text { if } \bar{\lambda}_{i_{2}}=\lambda_{i_{1}} .
$$

Proof: We need only to show that the set of vectors given by (28) satisfies (13). Then take differential of order $l$ on both sides of (27), and we obtain

$$
\begin{aligned}
& \left(\lambda \boldsymbol{I}_{n}-\boldsymbol{A}\right) \frac{\mathrm{d}^{l}}{\mathrm{~d} \lambda^{l}} \boldsymbol{N}(\lambda)+l \frac{\mathrm{d}^{l-1}}{\mathrm{~d} \lambda^{l-1}} \boldsymbol{N}(\lambda) \\
& +\lambda \boldsymbol{B} \frac{\mathrm{d}^{l}}{\mathrm{~d} \lambda^{l}} \widetilde{\boldsymbol{D}}(\lambda)+l \boldsymbol{B} \frac{\mathrm{d}^{l-1}}{\mathrm{~d} \lambda^{l-1}} \widetilde{\boldsymbol{D}}(\lambda)=0 .
\end{aligned}
$$

Substituting $\lambda$ by $\lambda_{i}$ and postmultiplying by a vector $\frac{1}{l !} f_{i j}^{k-1}$ on both sides of (29) gives

$$
\begin{aligned}
& \left(\lambda_{i} \boldsymbol{I}_{n}-\boldsymbol{A}\right) \frac{1}{l !} \frac{\mathrm{d}^{l}}{\mathrm{~d} \lambda^{l}}\left(N\left(\lambda_{i}\right)\right) f_{i j}^{k-1}+\lambda_{i} \boldsymbol{B} \frac{1}{l !} \frac{\mathrm{d}^{l}}{\mathrm{~d} \lambda^{l}}\left(\widetilde{\boldsymbol{D}}\left(\lambda_{i}\right)\right) f_{i j}^{k-1} \\
& =-\frac{1}{(l-1) !} \frac{\mathrm{d}^{l-1}}{\mathrm{~d} \lambda^{l-1}}\left(N\left(\lambda_{i}\right)\right) f_{i j}^{k-1}-\boldsymbol{B} \frac{1}{(l-1) !} \frac{\mathrm{d}^{l-1}}{\mathrm{~d} \lambda^{l-1}}\left(\widetilde{\boldsymbol{D}}\left(\lambda_{i}\right)\right) f_{i j}^{k-1}
\end{aligned}
$$

Summing up all the equations in (30) and using (28) we obtain

$\left(\lambda_{i} \boldsymbol{I}_{n}-\boldsymbol{A}\right) \mathbf{v}_{i j}^{k}+\lambda_{i} \boldsymbol{B} \mathbf{w}_{i j}^{k}=-\mathbf{v}_{i j}^{k-1}-\boldsymbol{B} \mathbf{w}_{i j}^{k-1}$,

$\mathbf{v}_{i j}^{0}=\boldsymbol{0}, \mathbf{w}_{i j}^{0}=\boldsymbol{0}, \forall i, j, k$,

where the assignable chains of right eigenvector generalized eigenvectors associated with the assigned eigenvalue $\lambda_{i}$ are given by

$$
\begin{aligned}
\mathbf{v}_{i j}^{k} & =\boldsymbol{N}\left(\lambda_{i}\right) f_{i j}^{k}+\frac{\mathrm{d}}{\mathrm{d} \lambda}\left(\boldsymbol{N}\left(\lambda_{i}\right)\right) f_{i j}^{k-1}+\ldots \\
& +\frac{1}{(k-1) !} \frac{\mathrm{d}^{k-1}}{\mathrm{~d} \lambda^{k-1}}\left(\boldsymbol{N}\left(\lambda_{i}\right)\right) f_{i j}^{1}, \mathbf{v}_{i j}^{0}=\boldsymbol{O} .
\end{aligned}
$$

Similarity, the gain-eigenvector product

$$
\begin{aligned}
\mathbf{w}_{i j}^{k} & =\widetilde{\boldsymbol{D}}\left(\lambda_{i}\right) f_{i j}^{k}+\frac{\mathrm{d}}{\mathrm{d} \lambda}\left(\widetilde{\boldsymbol{D}}\left(\lambda_{i}\right)\right) f_{i j}^{k-1}+\ldots \\
& +\frac{1}{(k-1) !} \frac{\mathrm{d}^{k-1}}{\mathrm{~d} \lambda^{k-1}}\left(\widetilde{\boldsymbol{D}}\left(\lambda_{i}\right)\right) f_{i j}^{1}, \mathbf{w}_{i j}^{0}=\mathbf{0} .
\end{aligned}
$$

Summing up, all the equations (28) hold.

Then, Theorems 1 and 2 give two complete and explicit parametric solutions with the complete and explicit freedom of eigenstructure assignment via state-derivative feedback. These solutions are expressed by the eigenvalues and a group of free parameter vectors, $f_{i j}^{k}$. By specially choosing the free parameter vectors in (18) and (28), solutions with desired properties can be obtained.

Remark 1: It should be noted that for the case of distinct eigenvalues $\left(m_{i}=q_{i}=1, s=n\right)$. Then, the computations of $\mathbf{v}_{i j}^{k}$ and $\mathbf{w}_{i j}^{k}$, taking the simple form, are given by:

$$
\left(\begin{array}{c}
\mathbf{v}_{i 1}^{1} \\
\mathbf{w}_{i 1}^{1}
\end{array}\right)=\left(\begin{array}{c}
\boldsymbol{Q}_{1}\left(\lambda_{i}\right) \\
\widetilde{\boldsymbol{Q}}_{2}\left(\lambda_{i}\right)
\end{array}\right)\left(\begin{array}{c}
f_{i 1}^{1} \\
\boldsymbol{0}
\end{array}\right)
$$

or

$$
\left(\begin{array}{c}
\mathbf{v}_{i 1}^{1} \\
\mathbf{w}_{i 1}^{1}
\end{array}\right)=\left(\begin{array}{c}
\boldsymbol{N}\left(\lambda_{i}\right) \\
\widetilde{\boldsymbol{D}}\left(\lambda_{i}\right)
\end{array}\right) f_{i 1}^{1}, i=1, \ldots, n .
$$

Remark 2: For the single-input system $(m=1)$, the parameter vectors $f_{i j}^{k}$ reduce to scalars and, accordingly, the solutions of (18) and (28) are the same (unique), regardless of the choice of $f_{i j}^{k}$. This leads to the well-known result that solution $K$ in this case is unique $[1,2]$.

The general expressions for the closed-loop eigenvectors and the feedback gains can immediately be written out as soon as the two polynomial matrix reductions (16) and (26) are carried out. These reductions can be completed by a series of simple elementary matrix transformations [6].

There are two methods for computing the polynomial matrices. The first is the Smith canonical form, which exploits the fact that for a controllable pair $(\boldsymbol{A}, \boldsymbol{B})$ the matrix $\left(\lambda \boldsymbol{I}_{n}-\boldsymbol{A}, \boldsymbol{B}\right)$ maintains full rank for all values of $\lambda$. The Smith canonical form constructs two unimodular matrices $\boldsymbol{P}(\lambda)$ and $\boldsymbol{Q}(\lambda)$ that diagonalized a given polynomial matrix as (16). Subject to the controllability of $(\boldsymbol{A}, \boldsymbol{B})$ the augmented matrix

$$
\boldsymbol{G}=\left(\begin{array}{ccc}
\boldsymbol{I}_{n} & \lambda \boldsymbol{I}_{n}-\boldsymbol{A} & \boldsymbol{B} \\
& 0 & \boldsymbol{I}_{n+m}
\end{array}\right),
$$

can be changed into the form of

$$
\boldsymbol{H}=\left(\begin{array}{ccc}
\boldsymbol{P}(\lambda) & \boldsymbol{0} & \boldsymbol{I}_{n} \\
\boldsymbol{0} & \boldsymbol{Q}(\lambda) &
\end{array}\right) .
$$

By applying a series of row elementary transformations within the upper $n$ rows and a series of column elementary transformations within the last $n+m$ columns, the matrices $\boldsymbol{P}(\lambda)$ and $\boldsymbol{Q}(\lambda)$ in the final transformed matrix $\boldsymbol{H}$ are unimodular and automatically satisfying (16). Consequently, we can partition $\boldsymbol{Q}(\lambda)$ to find $\boldsymbol{N}(\lambda)$ and $\boldsymbol{D}(\lambda)$ as

$$
Q(\lambda)=\left(\begin{array}{ll}
\times & N(\lambda) \\
\times & D(\lambda)
\end{array}\right) .
$$

The second approach uses the matrix fraction description (MFD). If all the elements of the matrix are proper rational polynomials, then the matrix may be factored as $N(\lambda) D^{-1}(\lambda)$. The elements of $\left(\lambda \boldsymbol{I}_{n}-\boldsymbol{A}\right)^{-1} \boldsymbol{B}$ are rational polynomials. Thus

$$
\left(\lambda \boldsymbol{I}_{n}-\boldsymbol{A}\right)^{-1} \boldsymbol{B}=\boldsymbol{N}(\lambda) \boldsymbol{D}^{-1}(\lambda) .
$$

The convenient solution can be found by inspection $\boldsymbol{N}(\lambda)=\left(\lambda \boldsymbol{I}_{n}-\boldsymbol{A}\right)^{-1} \boldsymbol{B}$ and $\boldsymbol{D}(\lambda)=\boldsymbol{I}_{n}$,

or

$\boldsymbol{N}(\lambda)=\operatorname{adj}\left(\lambda \boldsymbol{I}_{n}-\boldsymbol{A}\right) \boldsymbol{B}$ and $\boldsymbol{D}(\lambda)=\operatorname{det}\left(\lambda \boldsymbol{I}_{n}-\boldsymbol{A}\right) \boldsymbol{I}_{n}$,

where $\operatorname{adj}($.$) and \operatorname{det}($.$) represent, respectively, the adjoint and$ the determinant of matrix (.).

The Smith canonical form and MFD approaches both require symbolic manipulation to perform the Smith decomposition or matrix inversion. This presents no difficulty when working by hand or using a symbolic package such as Maple. 
Based on the discussion and analysis above, an algorithm for solving the eigenstructure assignment problem via state-derivative feedback can be given as follows:

\section{Algorithm}

Input Controllable real pair $(\boldsymbol{A}, \boldsymbol{B})$, where matrix $\boldsymbol{A} \in \mathrm{R}^{n \times n}$ is nonsingular, and a set of $n$ self-conjugate complex numbers $\left\{\lambda_{1}, \ldots, \lambda_{n}\right\}$.

Step 1 Construct the right coprime matrix polynomials $N(\lambda)$ and $\boldsymbol{D}(\lambda)$ by applying the method presented in this section.

Step 2 Choose arbitrary parameter vectors $f_{i j}^{k} \in \mathrm{C}^{m}(i=1, \ldots$, $\left.s, j=1, \ldots, q_{i}, k=1, \ldots, p_{i j}\right)$ in such a way that $\bar{f}_{i_{2} j}^{k}=f_{i_{1} j}^{k}$ implies $\bar{\lambda}_{i_{2}}=\lambda_{i_{1}}$.

Step 3 Calculate the right eigenvectors $\mathbf{v}_{i j}^{k} \in \mathrm{C}^{n}(i=1, \ldots, s$, $\left.j=1, \ldots, q_{i}, k=1, \ldots, p_{i j}\right)$ using (28). If the eigenvectors matrix $V$ is singular, then return to Step 2 and select different parameters $f_{i j}^{k}$, until $\boldsymbol{V}$ is nonsingular.

Step 4 Compute the gain-eigenvectors $\mathbf{w}_{i j}^{k} \in \mathrm{C}^{m}(i=1, \ldots, s$, $\left.j=1, \ldots, q_{i}, k=1, \ldots, p_{i j}\right)$ using (28), and construct matrix $\boldsymbol{W}$.

Step 5 Compute the real derivative feedback gain matrix using, $K=W V^{-1}$.

From the above results we can observe that the system poles can always be assigned by a state-derivative feedback controller for any controllable system if and only if the open-loop system matrix $\boldsymbol{A}$ is nonsingular. In the case of single-input, $m=1$, there only at most one solution. In the case of multi-input, $1<m \leq n$, the solution is generally non-unique, and extra conditions must be imposed to specify a solution. The extra freedom can be used to give the closed-loop system other desirable properties. The extra freedom can be used in different ways, for example to decrease the norm of the feedback gain matrix or to improve the condition of the eigenvectors of the closed-loop matrix. Additionally, it increases the robustness of the closed-loop system against the system parameter perturbation. This issue becomes very important when the system model is not sufficiently precise or the system is subject to parameter uncertainty. Then the feedback gain matrix is parameterized directly in terms of the eigenstructure of the closed-loop system, which can be selected to ensure robustness by exploiting freedom of these parameters.

Eigenstructure assignment is a very flexible technique. It provides access to all the available design freedom. The drawback of eigenstructure assignment is that it has no inherent mechanism for insuring robustness and can assign a robust solution as easily as a catastrophically unrobust solution. Then the optimization techniques are used with the objective of finding the optimum design vectors $f_{i j}^{k}$ so that the closed-loop system is robust to parameter variations.

\section{Illustrative examples}

In this section, numerical examples are used to illustrate the feasibility and effectiveness of the proposed eigenstructure assignment technique via state-derivative feedback.

\section{Example 1:}

Consider a controllable, time-invariant, multi-input linear system,

$$
\dot{\boldsymbol{x}}(t)=\left(\begin{array}{lll}
0 & 1 & 0 \\
0 & 0 & 1 \\
1 & 0 & 1
\end{array}\right) \boldsymbol{x}(t)+\left(\begin{array}{ll}
0 & 0 \\
0 & 1 \\
1 & 0
\end{array}\right) \boldsymbol{u}(t) .
$$

A pair of right matrix polynomials $N(\lambda)$ and $D(\lambda)$ satisfying (25) and $\widetilde{\boldsymbol{D}}(\lambda)$ can be found as:

$$
\begin{aligned}
& \boldsymbol{N}(\lambda)=\left(\begin{array}{cc}
1 & 0 \\
\lambda & 0 \\
0 & -1
\end{array}\right), \boldsymbol{D}(\lambda)=\left(\begin{array}{cc}
-1 & -\lambda+1 \\
\lambda^{2} & 1
\end{array}\right) \\
& \text { and } \widetilde{\boldsymbol{D}}(\lambda)=\left(\begin{array}{cc}
1 / \lambda & 1-1 / \lambda \\
-\lambda & -1 / \lambda
\end{array}\right) .
\end{aligned}
$$

In the following, we consider the assignment of three different cases:

Case 1: The desired closed-loop eigenvalues are selected as $\{-1,-2$ and -3$\}$.

Then, the closed-loop eigenvector matrix $\boldsymbol{V}$, and the corresponding matrix $\boldsymbol{W}$, can be written as

$\boldsymbol{V}=\left[\boldsymbol{N}\left(\lambda_{1}\right) f_{11}^{1}, \boldsymbol{N}\left(\lambda_{2}\right) f_{21}^{1}, \boldsymbol{N}\left(\lambda_{3}\right) f_{31}^{1}\right]$ and

$\boldsymbol{W}=\left[\widetilde{\boldsymbol{D}}\left(\lambda_{1}\right) f_{11}^{1}, \widetilde{\boldsymbol{D}}\left(\lambda_{2}\right) f_{21}^{1}, \widetilde{\boldsymbol{D}}\left(\lambda_{3}\right) f_{31}^{1}\right]$.

Specially choosing

$$
f_{11}^{1}=f_{31}^{1}=[1,0]^{\mathrm{T}} \text { and } f_{21}^{1}=[0,1]^{\mathrm{T}} .
$$

Then

$$
\boldsymbol{V}=\left(\begin{array}{ccc}
1 & 0 & 1 \\
-1 & 0 & -3 \\
0 & -1 & 0
\end{array}\right) \text { and } \boldsymbol{W}=\left(\begin{array}{ccc}
-1 & 1.5 & -1 / 3 \\
1 & 0.5 & 3
\end{array}\right)
$$

Finally the state-derivative gain matrix is

$$
\boldsymbol{K}=\boldsymbol{W} \boldsymbol{V}^{-1}=\left(\begin{array}{ccc}
-4 / 3 & -1 / 3 & -1.5 \\
0 & -1 & -0.5
\end{array}\right) \text {. }
$$

Case 2: The desired closed-loop poles are $\{-2$ and $-3 \pm i\}$.

Choosing

$$
f_{11}^{1}=[0,1]^{\mathrm{T}} \text { and } f_{21}^{1}=f_{31}^{1}=[1,0]^{\mathrm{T}} .
$$

Then

$$
\boldsymbol{V}=\left(\begin{array}{ccc}
0 & 1 & 1 \\
0 & -3-i & -3+i \\
-1 & 0 & 0
\end{array}\right) \text { and } \boldsymbol{W}=\left(\begin{array}{ccc}
1.5 & -0.3+i & -0.3-i \\
0.5 & 3+i & 3-i
\end{array}\right)
$$

The gain matrix is

$$
\boldsymbol{K}=\boldsymbol{W} \boldsymbol{V}^{-1}=\left(\begin{array}{ccc}
-0.6 & -0.1 & -1.5 \\
0 & -1 & -0.5
\end{array}\right) \text {. }
$$

Case 3: The desired eigenvalues are $\{-1,-1$ and -3$\}$.

$$
\text { Then }
$$$$
\boldsymbol{V}=\left[\boldsymbol{N}\left(\lambda_{1}\right) f_{11}^{1}, \frac{\mathrm{d}}{\mathrm{d} \lambda}\left(\boldsymbol{N}\left(\lambda_{1}\right)\right) f_{11}^{1}+\mathbf{N}\left(\lambda_{1}\right) f_{11}^{2}, \boldsymbol{N}\left(\lambda_{2}\right) f_{21}^{1}\right] \text { and }
$$$$
W=\left[\widetilde{\boldsymbol{D}}\left(\lambda_{1}\right) f_{11}^{1}, \frac{\mathrm{d}}{\mathrm{d} \lambda}\left(\widetilde{\boldsymbol{D}}\left(\lambda_{1}\right)\right) f_{11}^{1}+\widetilde{\boldsymbol{D}}\left(\lambda_{1}\right) f_{11}^{2}, \widetilde{\boldsymbol{D}}\left(\lambda_{2}\right) f_{21}^{1}\right] \text {. }
$$

Choosing

$$
f_{11}^{1}=f_{21}^{1}=[1,0]^{\mathrm{T}} \text { and } f_{11}^{2}=[0,1]^{\mathrm{T}} .
$$


We have

$\boldsymbol{V}=\left(\begin{array}{ccc}1 & 0 & 1 \\ -2 & 0 & -3 \\ 0 & -1 & 0\end{array}\right)$ and $\boldsymbol{W}=\left(\begin{array}{ccc}-0.5 & 1.5 & -1 / 3 \\ 2 & 0.5 & 3\end{array}\right)$.

Therefore

$\boldsymbol{K}=\boldsymbol{W} \boldsymbol{V}^{-1}=\left(\begin{array}{ccc}-0.8333 & -0.1667 & -1.5 \\ 0 & -1 & -0.5\end{array}\right)$.

\section{Example 2:}

Consider a controllable, multi-input, linear system

$$
\dot{x}(t)=\left(\begin{array}{ccc}
-2.5 & 0.5 & 0 \\
0.5 & -2.5 & 2 \\
0 & 2 & -2
\end{array}\right) \boldsymbol{x}(t)+\left(\begin{array}{ll}
1 & 0 \\
0 & 0 \\
0 & 1
\end{array}\right) \boldsymbol{u}(t) .
$$

A pair of right matrix polynomials $N(\lambda)$ and $\boldsymbol{D}(\lambda)$ can be obtained as:

$\boldsymbol{N}(\lambda)=\left(\begin{array}{cc}2 \lambda+5 & -4 \\ 1 & 0 \\ 0 & 1\end{array}\right)$ and
$\boldsymbol{D}(\lambda)=\left(\begin{array}{cc}2(\lambda+2.5)^{2}-0.5 & -4 \lambda-10 \\ -2 & \lambda+2\end{array}\right)$.

In the following, we consider the assignment of three different cases:

Case 1: The desired closed-loop eigenvalues are selected as $\{-1,-2$ and -3$\}$.

Specially choosing

$f_{11}^{1}=f_{31}^{1}=[1,0]^{\mathrm{T}}$ and $f_{21}^{1}=[0,1]^{\mathrm{T}}$.

Then

$\boldsymbol{V}=\left(\begin{array}{ccc}3 & -4 & -1 \\ 1 & 0 & 1 \\ 0 & 1 & 0\end{array}\right)$ and $\boldsymbol{W}=\left(\begin{array}{ccc}4 & -1 & 0 \\ -2 & 0 & -0.6667\end{array}\right)$.

The gain matrix is

$\boldsymbol{K}=\boldsymbol{W} \boldsymbol{V}^{-1}=\left(\begin{array}{ccc}1 & 1 & 3 \\ -0.3334 & -1 & -1.3334\end{array}\right)$.

Case 2: The desired closed-loop eigenvalues are $\{-2$ and $-3 \pm i\}$.

Choosing

$$
f_{11}^{1}=[0,1]^{\mathrm{T}} \text { and } f_{21}^{1}=f_{31}^{1}=[1,0]^{\mathrm{T}} .
$$

Then

$$
\begin{aligned}
& \boldsymbol{V}=\left(\begin{array}{ccc}
-4 & -1-2 i & -1+2 i \\
0 & 1 & 1 \\
1 & 0 & 0
\end{array}\right), \\
& \boldsymbol{W}=\left(\begin{array}{ccc}
-1 & -0.4+0.8 i & -0.4-0.8 i \\
0 & -0.6+0.2 i & -0.6-0.2 i
\end{array}\right) .
\end{aligned}
$$

The gain matrix

$$
\boldsymbol{K}=\boldsymbol{W} \boldsymbol{V}^{-1}=\left(\begin{array}{rrr}
-0.4 & -0.8 & -2.6 \\
-0.1 & -0.7 & -0.4
\end{array}\right) .
$$

Case 3: The desired closed-loop eigenvalues are $\{-2,-2$ and $-3\}$.
Choosing

$$
f_{11}^{1}=f_{21}^{1}=[1,0]^{\mathrm{T}} \text { and } f_{11}^{2}=[0,1]^{\mathrm{T}} .
$$

Then

$$
\boldsymbol{V}=\left(\begin{array}{ccc}
1 & -4 & -1 \\
1 & 0 & 1 \\
0 & 1 & 0
\end{array}\right) \text { and } \boldsymbol{W}=\left(\begin{array}{ccc}
0 & -1 & 0 \\
-1 & 0 & -0.6667
\end{array}\right)
$$

Therefore

$$
\boldsymbol{K}=\boldsymbol{W} \boldsymbol{V}^{-1}=\left(\begin{array}{ccc}
0 & 0 & -1 \\
-0.1667 & -0.8333 & -0.6667
\end{array}\right)
$$

\section{Conclusions}

In this paper, two complete parametric approaches for solving the eigenstructure assignment problem via state-derivative feedback are proposed. The necessary conditions to ensure solvability are that the system is completely controllable and the open-loop system matrix is nonsingular. The main result of this work is an efficient computational algorithm for solving the eigenstructure assignment problem of a linear system via state-derivative feedback. This parametric solution describes the available degrees of freedom offered by the state-derivative feedback in selecting the associated eigenvectors from an admissible class. The extra degrees of freedom on the choice of feedback gains are exploited to further improve the closed-loop robustness against perturbation. The main contribution of the present work is a compact parametric expression for the feedback controller gain matrix explicitly characterized by a set of free parameter vectors. The principle benefits of the explicit characterization of parametric class of feedback controllers lie in the ability to directly accommodate various different design criteria.

\section{References}

[1] Abdelaziz. T. H. S., Valášek, M.: "Pole-Placement for SISO Linear Systems by State-Derivative Feedback.” IEE Proceeding Part D: Control Theory \& Applications, 151, 4, 377-385, 2004.

[2] Abdelaziz, T. H. S., Valášek, M.: "A Direct Algorithm for Pole Placement by State-Derivative Feedback for Single-Input Linear Systems.” Acta Polytechnica, Vol. 43 (2003), No. 6, p. 52-60.

[3] Abdelaziz, T. H. S., Valášek, M.: "A Direct Algorithm for Pole Placement by State-Derivative Feedback for Multi-Input Linear Systems - Nonsingular Case.” (Accepted in Kybernetika, 2004).

[4] Abdelaziz, T. H. S., Valášek, M.: "Eigenstructure Assignment by State-Derivative and Partial Output-Derivative Feedback for Linear Time-Invariant Control Systems." Acta Polytechnica, Vol. 44 (2004), No. 4, p. 54-60.

[5] Abdelaziz, T. H. S., Valášek, M.: "Parametric Solutions of Eigenstructure Assignment by State-Derivative Feedback for Linear Control Systems." Proceedings of Interaction and Feedbacks 2003, UT AV CR, Praha, 2003, p. 5-12.

[6] Duan, G. R: "Solutions of the Matrix Equation AV+BW $=\mathrm{VF}$ and Their Application to Eigenstructure Assign- 
ment In Linear Systems." IEEE Transactions on Automatic Control, Vol. 38 (1993), No. 2. p. 276-280.

[7] Fahmy, M. M., Tantawy, H. S.: "Eigenstructure Assignment via Linear State Feedback Control." International Journal of Control, Vol. 40 (1984), No. 1, p. 161-178.

[8] Fahmy, M. M., O'Reilly, J.: "Eigenstructure Assignment in Linear Multivariable Systems: a Parametric Solution.” IEEE Transactions on Automatic Control, Vol. 28 (1983), No. 10, p. 990-994.

[9] Fahmy, M. M., O’Reilly, J.: “On Eigenstructure Assignment in Linear Multivariable Systems." IEEE Transactions on Automatic Control, Vol. 27 (1982), No. 3, p. 690-693.

[10] Clarke, T., Griffin, S. J., Ensor, J.: “A Polynomial Approach to Eigenstructure Assignment Using Projection with Eigenvalue Trade-Off." International Journal of Control, Vol. 76 (2003), No. 4, p. 403-423.

[11] Kautsky, J., Nichols, N. K., Van Dooren, P.: "Robust Pole Assignment in Linear State Feedback.” International Journal of Control, Vol. 41 (1985), p. 1129-1155.

[12] Preumont, A., Loix, N., Malaise, D., Lecrenier, O.: “Active Damping of Optical Test Benches with Acceleration Feedback." Machine Vibration, Vol. 2 (1993), p. 119-124.

[13] Preumont, A.: Vibration Control of Active Structures, Kluwer, 1998.

[14] Bayon de Noyer, M. P., Hanagud, S. V.: "Single Actuator and Multi-Mode Acceleration Feedback Control." Adaptive Structures and Material Systems, ASME, AD, Vol. 54 (1997), p. 227-235.

[15] Bayon de Noyer, M. P., Hanagud, S. V.: "A Comparison of H2 Optimized Design and Cross-Over Point Design for Acceleration Feedback Control." Proceedings of $39^{\text {th }}$ AIAA/ASME/ ASCE/AHS, Structures, Structural
Dynamics and Materials Conference, Vol. 4 (1998), p. 3250-3258.

[16] Olgac, N., Elmali, H., Hosek, M., Renzulli, M.:“ Active Vibration Control of Distributed Systems Using Delayed Resonator with Acceleration Feedback." Transactions of ASME Journal of Dynamic Systems, Measurement and Control, Vol. 119 (1997), p. 380.

[17] Kejval, J., Sika, Z., Valášek, M.: “Active Vibration Suppression of a Machine." Proceedings of Interaction and Feedbacks 2000, UT AV CR, Praha, 2000, p. 75-80.

[18] Deur, J., Peric, N.: A Comparative Study of Servosystems with Acceleration Feedback." Proceedings of the $35^{\text {th }}$ IEEE Industry Applications Conference, Roma, Italy, Vol. 2 (2000), p. 1533-1540.

[19] Ellis, G.: "Cures for Mechanical Resonance in Industrial Servo Systems.” Proceedings of PCIM 2001 Conference, Nuremberg, 2001.

Doc. Taha H. S. Abdelaziz

e-mail: tahahelmy@yahoo.com

Department of Mechanical Engineering

Faculty of Engineering

Helwan University

1 Sherif Street, Helwan, Cairo, Egypt

Doc. Ing. Michael Valášek, DrSc.

e-mail:valasek@fsik.cvut.cz

Department of Mechanics

Czech Technical University in Prague

Faculty of Mechanical Engineering

Karlovo nám. 13

12135 Praha 2, Czech Republic 